\title{
A Nonlinear Case of the 1-D Backward Heat Problem: Regularization and Error Estimate
}

\author{
Dang Duc Trong, Pham Hoang Quan, Tran Vu Khanh \\ and Nguyen Huy Tuan
}

\begin{abstract}
We consider the problem of finding, from the final data $u(x, T)=\varphi(x)$, the temperature function $u(x, t), x \in(0, \pi), t \in[0, T]$ satisfies the following nonlinear system

$$
\begin{aligned}
u_{t}-u_{x x} & =f(x, t, u(x, t)), & & (x, t) \in(0, \pi) \times(0, T) \\
u(0, t) & =u(\pi, t)=0, & & t \in(0, T) .
\end{aligned}
$$

The nonlinear problem is severely ill-posed. We shall improve the quasi-boundary value method to regularize the problem and to get some error estimates. The approximation solution is calculated by the contraction principle. A numerical experiment is given.
\end{abstract}

Keywords. Backward heat problem, nonlinearly Ill-posed problem, quasi-boundary value methods, quasi-reversibility methods, contraction principle

Mathematics Subject Classification (2000). Primary 35K05, secondary 35K99, 47J06, 47H10

\section{Introduction}

Let $\mathrm{T}$ be a positive number, we consider the problem of finding the temperature $u(x, t),(x, t) \in(0, \pi) \times[0, T]$ such that

$$
\begin{aligned}
u_{t}-u_{x x} & =f(x, t, u(x, t)), & & (x, t) \in(0, \pi) \times(0, T) \\
u(0, t) & =u(\pi, t)=0, & & t \in(0, T) \\
u(x, T) & =\varphi(x), & & x \in(0, \pi),
\end{aligned}
$$

where $\varphi(x), f(x, t, z)$ are given. The problem is called the backward heat problem, the backward Cauchy problem or the final value problem.

HoChiMinh City National University, Department of Mathematics and Informatics, 227 Nguyen Van Cu, Q. 5, HoChiMinh City, VietNam; tquan@pmail.vnn.vn 
As is known, the nonlinear problem is severely ill-posed, i.e., solutions do not always exist, and in the case of existence, these do not depend continuously on the given data. In fact, from small noise contaminated physical measurements, the corresponding solutions have large errors. It makes difficult to numerical calculations. Hence, a regularization is in order. The linear case was studied extensively in the last four decades by many methods. The literature related to the problem is impressive (see, e.g., $[3,4,7]$ and the references therein). In the pioneering work [7] in 1967, the authors presented, in a heuristic way, the quasireversibility method. They approximated the problem by adding a "corrector" into the main equation. In fact, they considered the problem

$$
\begin{aligned}
u_{t}+A u-\epsilon A^{*} A u & =0, \quad t \in[0, T] \\
u(T) & =\varphi .
\end{aligned}
$$

The stability magnitude of the method is of order $e^{c \epsilon^{-1}}$. In $[1,12]$, the problem is approximated with

$$
\begin{aligned}
u_{t}+A u+\epsilon A u_{t} & =0, \quad t \in[0, T] \\
u(T) & =\varphi .
\end{aligned}
$$

The method is useful if we cannot construct clearly the operator $A^{*}$. However, the stability order in the case is quite as large as that in the original quasireversibility methods. In [10], using the method, so-called, of stabilized quasi reversibility, the author approximated the problem with

$$
\begin{aligned}
u_{t}+f(A) u & =0, \quad t \in[0, T] \\
u(T) & =\varphi .
\end{aligned}
$$

He shows that, with appropriate conditions on the "corrector" $f(A)$, the stability magnitude of the method is of order $c \epsilon^{-1}$.

Sixteen years after the work by Lattes-Lions, in 1983, Showalter presented the quasi-boundary value method. He considered the problem

$$
\begin{aligned}
u_{t}-A u(t) & =B u(t), \quad t \in[0, T] \\
u(0) & =\varphi
\end{aligned}
$$

and approximated the problem with

$$
\begin{aligned}
u_{t}-A u(t) & =B u(t), \quad t \in[0, T] \\
u(0)+\epsilon u(T) & =\varphi .
\end{aligned}
$$

According to him, this method gives a better stability estimate than the other discussed methods. Clark and Oppenheimer, in their paper [4], used the quasiboundary value method to regularize the backward problem with

$$
\begin{aligned}
u_{t}+A u(t) & =0, \quad t \in[0, T] \\
u(T)+\epsilon u(0) & =\varphi .
\end{aligned}
$$


The authors show that the stability estimate of the method is of order $\epsilon^{-1}$. Very recently, in [6], the quasi-boundary method was used to solve a backward heat equation with an integral boundary condition.

Although we have many works on the linear case of the backward problem, the literature of the nonlinear case is quite scarce. Very recently, in [11], the authors tranform the problem into the one of minimizing an appropriate functional. However, a sharp error estimate and an effective method of calculation are not given in [11].

Informally, problem (1)-(3) can be transformed to an integral equation having the form

$$
u(x, t)=\sum_{n=1}^{\infty}\left[e^{(T-t) n^{2}} \varphi_{n}-\int_{t}^{T} e^{(s-t) n^{2}} f_{n}(u)(s) d s\right] \sin n x
$$

where $\varphi(x)=\sum_{n=1}^{\infty} \varphi_{n} \sin n x, f(u)(x, t)=\sum_{n=1}^{\infty} f_{n}(u)(t) \sin n x$ are the expansion of $\varphi$ and $f(u)$, respectively. The terms $e^{(T-t) n^{2}}, e^{(s-t) n^{2}}(n$ large) are the unstability cause. Hence, to regularize the problem, we have to replace the terms by better terms. Naturally, we shall replace two terms by

$$
\frac{e^{-t n^{2}}}{\alpha_{n}(\varepsilon, t)+e^{-T n^{2}}}, \quad \frac{e^{-t n^{2}}}{\beta_{n}(\varepsilon, t, s)+e^{-s n^{2}}},
$$

where $\alpha_{n}, \beta_{n}$ are positive functions satisfying

$$
\lim _{\varepsilon \downarrow 0} \alpha_{n}(\varepsilon, t)=\lim _{\varepsilon \downarrow 0} \beta_{n}(\varepsilon, t, s)=0 .
$$

Many versions of $\alpha_{n}, \beta_{n}$ are suggested from the quasi-type methods discussed above.

In the present paper, we shall use an association of the quasi-reversibility method and the quasi-boundary value method to regularize our problem. In fact, we approximate problem (1)-(3) by the following problem:

$$
\begin{aligned}
u_{t}^{\epsilon}-u_{x x}^{\epsilon} & =\sum_{n=1}^{\infty} \frac{e^{-t n^{2}}}{\epsilon^{\frac{t}{T}}+e^{-t n^{2}}} f_{n}\left(u^{\epsilon}\right)(t) \sin n x, \quad(x, t) \in(0, \pi) \times(0, T) \\
u^{\epsilon}(0, t) & =u^{\epsilon}(\pi, t)=0, \quad t \in[0, T] \\
\epsilon u^{\epsilon}(x, 0) & +u^{\epsilon}(x, T) \\
& =\varphi(x)-\sum_{n=1}^{\infty}\left(\int_{0}^{T} \frac{\epsilon}{\epsilon^{\frac{s}{T}}+e^{-s n^{2}}} f_{n}\left(u^{\epsilon}\right)(s) d s\right) \sin n x, \quad x \in[0, \pi]
\end{aligned}
$$

where $0<\epsilon<1, f_{n}(u)(t)=\frac{2}{\pi}\langle f(x, t, u(x, t)), \sin n x\rangle$ and $\langle\cdot, \cdot\rangle$ is the inner product in $L^{2}(0, \pi)$. We shall prove that, the (unique) solution $u^{\epsilon}$ of $(4)-(6)$ 
satisfies the following equality:

$$
u^{\epsilon}(x, t)=\sum_{n=1}^{\infty}\left(\frac{e^{-t n^{2}}}{\epsilon+e^{-T n^{2}}} \varphi_{n}-\int_{t}^{T} \frac{e^{-t n^{2}}}{\epsilon^{\frac{s}{T}}+e^{-s n^{2}}} f_{n}\left(u^{\epsilon}\right)(s) d s\right) \sin n x,
$$

where $\varphi_{n}=\frac{2}{\pi}\langle\varphi(x), \sin n x\rangle$.

The remainder of the paper is divided into three sections. In Section 2, we shall show that $(4)-(6)$ is well posed and that the solution $u^{\epsilon}(x, t)$ satisfies (7). Then, in Section 3, we estimate the error between an exact solution $u_{0}$ of problem (1)-(3) and the approximation solution $u^{\epsilon}$. In fact, we shall prove that

$$
\left\|u^{\epsilon}(\cdot, t)-u_{0}(\cdot, t)\right\| \leq C \epsilon^{\frac{t}{T}}
$$

and that there is a $t_{\epsilon}>0$ such that

$$
\left\|u^{\epsilon}\left(\cdot, t_{\epsilon}\right)-u_{0}(\cdot, 0)\right\| \leq \sqrt[4]{8} C \sqrt[4]{T}\left(\ln \left(\frac{1}{\epsilon}\right)\right)^{-\frac{1}{4}}
$$

where $\|\cdot\|$ is the norm in $L^{2}(0, \pi)$ and $C$ depends on $u_{0}$ and $f$. Finally, a numerical experiment will be given in Section 4 .

\section{The well-posedness of problem (4)-(6)}

In the section, we shall study the existence, the uniqueness and the stability of a (weak) solution of problem (4)-(6). In fact, one has

Theorem 2.1. Let $\varphi \in L^{2}(0, \pi)$ and let $f \in L^{\infty}([0, \pi] \times[0, T] \times R)$ satisfy

$$
|f(x, y, w)-f(x, y, v)| \leq k|w-v|
$$

for $a k>0$ independent of $x, y, w, v$. Then problem (4)-(6) has uniquely a weak solution $u^{\epsilon} \in C\left([0, T] ; L^{2}(0, \pi)\right) \cap L^{2}\left(0, T ; H_{0}^{1}(0, \pi)\right) \cap C^{1}\left(0, T ; H_{0}^{1}(0, \pi)\right)$ satisfying (7). The solution depends continuously on $\varphi$ in $C\left([0, T] ; L^{2}(0, \pi)\right)$.

Proof. The proof is divided into three steps. In Step 1, we shall prove that problem (4)-(6) is equivalence to problem (7). In Step 2, we prove the existence and the uniqueness of a solution of (7). Finally in Step 3, the stability of the solution is given.

Step 1. Prove that (4)-(6) is equivalence (7). We divide this step into two parts.

Part A. If $u^{\epsilon} \in C\left([0, T] ; L^{2}(0, \pi)\right)$ satisfies $(7)$, then $u^{\epsilon}$ is solution of (4)-(6). 
For $0 \leq t \leq T$, we have

$$
u^{\epsilon}(x, t)=\sum_{n=1}^{\infty}\left(\frac{e^{-t n^{2}}}{\epsilon+e^{-T n^{2}}} \varphi_{n}-\int_{t}^{T} \frac{e^{-t n^{2}}}{\epsilon^{\frac{s}{T}}+e^{-s n^{2}}} f_{n}\left(u^{\epsilon}\right)(s) d s\right) \sin n x
$$

where $u^{\epsilon} \in C\left([0, T] ; L^{2}(0, \pi) \cap C^{1}\left((0, T) ; H_{0}^{1}(0, \pi)\right) \cap L^{2}\left(0, T ; H_{0}^{1}(0, \pi)\right)\right)$ can be verified directly. In fact, $\left.u^{\epsilon} \in C^{\infty}\left((0, T] ; H_{0}^{1}(0, \pi)\right)\right)$. Moreover, one has

$$
\begin{aligned}
& u_{t}^{\epsilon}(x, t)=\sum_{n=1}^{\infty}\left(\frac{-n^{2} e^{-t n^{2}}}{\epsilon+e^{-T n^{2}}} \varphi_{n}-\int_{t}^{T} \frac{-n^{2} e^{-t n^{2}}}{\epsilon^{\frac{s}{T}}+e^{-s n^{2}}} f_{n}\left(u^{\epsilon}\right)(s) d s\right) \sin n x \\
& +\sum_{n=1}^{\infty} \frac{e^{-t n^{2}}}{\epsilon^{\frac{t}{T}}+e^{-t n^{2}}} f_{n}\left(u^{\epsilon}\right)(t) \sin n x \\
& =-\frac{2}{\pi} \sum_{n=1}^{\infty} n^{2}\left\langle u^{\epsilon}(x, t), \sin n x\right\rangle \sin n x \\
& +\sum_{n=1}^{\infty}\left(\frac{e^{-t n^{2}}}{\epsilon^{\frac{t}{T}}+e^{-t n^{2}}} f_{n}\left(u^{\epsilon}\right)(t)\right) \sin n x \\
& =u_{x x}^{\epsilon}(x, t)+\sum_{n=1}^{\infty}\left(\frac{e^{-t n^{2}}}{\epsilon^{\frac{t}{T}}+e^{-t n^{2}}} f_{n}\left(u^{\epsilon}\right)(t)\right) \sin n x
\end{aligned}
$$

and

$$
\epsilon u^{\epsilon}(x, 0)+u^{\epsilon}(x, T)=\varphi-\sum_{n=1}^{\infty}\left(\int_{0}^{T} \frac{\epsilon}{\epsilon^{\frac{s}{T}}+e^{-s n^{2}}} f_{n}\left(u^{\epsilon}\right)(s) d s\right) \sin n x .
$$

So $u^{\epsilon}$ is the solution of (4)-(6).

Part B. If $u^{\epsilon}$ satisfies (4)-(6), then $u^{\epsilon}$ is a solution of (7).

In fact, taking the inner product of the equation (4) with respect to $\sin n x$ we get in view of (4)

$$
\frac{d}{d t} u_{n}^{\epsilon}(t)+n^{2} u_{n}^{\epsilon}(t)=\frac{e^{-t n^{2}}}{\epsilon^{\frac{t}{T}}+e^{-t n^{2}}} f_{n}\left(u^{\epsilon}\right)(t)
$$

where we recall that

$$
u_{n}^{\epsilon}(t)=\frac{2}{\pi}\left\langle u^{\epsilon}(x, t), \sin n x\right\rangle, f_{n}\left(u^{\epsilon}\right)(t)=\frac{2}{\pi}\left\langle f\left(x, t, u^{\epsilon}(x, t)\right), \sin n x\right\rangle .
$$

It follows that

$$
u_{n}^{\epsilon}(t)=e^{-t n^{2}} u_{n}^{\epsilon}(0)+\int_{0}^{t} e^{-(t-s) n^{2}} f_{n}\left(u^{\epsilon}\right)(s) d s .
$$


Hence, we have the Fourier expansion

$$
\begin{aligned}
u^{\epsilon}(x, t) & =\sum_{n=1}^{\infty}\left(e^{-t n^{2}} u_{n}^{\epsilon}(0)+\int_{0}^{t} e^{-(t-s) n^{2}} \frac{e^{-s n^{2}}}{\epsilon^{\frac{s}{T}}+e^{-s n^{2}}} f_{n}\left(u^{\epsilon}\right)(s) d s\right) \sin n x \\
& =\sum_{n=1}^{\infty}\left(e^{-t n^{2}} u_{n}^{\epsilon}(0)+\int_{0}^{t} \frac{e^{-t n^{2}}}{\epsilon^{\frac{s}{T}}+e^{-s n^{2}}} f_{n}\left(u^{\epsilon}\right)(s) d s\right) \sin n x
\end{aligned}
$$

Hence

$$
u^{\epsilon}(x, T)=\sum_{n=1}^{\infty}\left(e^{-T n^{2}} u_{n}^{\epsilon}(0)+\int_{0}^{T} \frac{e^{-T n^{2}}}{\epsilon^{\frac{s}{T}}+e^{-s n^{2}}} f_{n}\left(u^{\epsilon}\right)(s) d s\right) \sin n x .
$$

Substituting (16) into (6) gives

$$
\sum_{n=1}^{\infty}\left(\left(\epsilon+e^{-T n^{2}}\right) u_{n}^{\epsilon}(0)\right) \sin n x=\varphi-\sum_{n=1}^{\infty}\left(\int_{0}^{T} \frac{\epsilon+e^{-T n^{2}}}{\epsilon^{\frac{s}{T}}+e^{-s n^{2}}} f_{n}\left(u^{\epsilon}\right)(s) d s\right) \sin n x .
$$

We obtain

$$
u_{n}^{\epsilon}(0)=\frac{1}{\epsilon+e^{-T n^{2}}} \varphi_{n}-\int_{0}^{T} \frac{1}{\epsilon^{\frac{s}{T}}+e^{-s n^{2}}} f_{n}\left(u^{\epsilon}\right)(s) d s .
$$

Replacing (17) in (15), we receive (7). This completes the proof of Step 1.

Step 2. The existence and the uniqueness of solution of (7).

Put

$$
G(w)(x, t)=\varphi(x, t)-\sum_{n=1}^{\infty} \int_{t}^{T} \frac{e^{-t n^{2}}}{\epsilon^{\frac{s}{T}}+e^{-s n^{2}}} f_{n}(w)(s) d s \sin n x
$$

for $w \in C\left([0, T] ; L^{2}(0, \pi)\right)$, where $\varphi(x, t)=\sum_{n=1}^{\infty} \frac{e^{-t n^{2}}}{\epsilon+e^{-T n^{2}}} \varphi_{n} \sin n x$. We claim that, for every $w, v \in C\left([0, T] ; L^{2}(0, \pi)\right), m \geq 1$, we have

$$
\left\|G^{m}(w)(\cdot, t)-G^{m}(v)(\cdot, t)\right\|^{2} \leq\left(\frac{k}{\epsilon}\right)^{2 m} \frac{(T-t)^{m} C^{m}}{m !}|\|w-v\||^{2}
$$

where $C=\max \{T, 1\}$ and $|\|\cdot\||$ is the supremum norm in $C\left([0, T] ; L^{2}(0, \pi)\right)$. We shall prove the latter inequality by induction. 
For $m=1$, we have

$$
\begin{aligned}
\| G(w)(\cdot, t) & -G(v)(\cdot, t) \|^{2} \\
& =\frac{\pi}{2} \sum_{n=1}^{\infty}\left[\int_{t}^{T} \frac{e^{-t n^{2}}}{\epsilon^{\frac{s}{T}}+e^{-s n^{2}}}\left(f_{n}(w)(s)-f_{n}(v)(s)\right) d s\right]^{2} \\
& \leq \frac{\pi}{2} \sum_{n=1}^{\infty} \int_{t}^{T}\left(\frac{e^{-t n^{2}}}{\epsilon^{\frac{s}{T}}+e^{-s n^{2}}}\right)^{2} d s \int_{t}^{T}\left(f_{n}(w)(s)-f_{n}(v)(s)\right)^{2} d s \\
& \leq \frac{\pi}{2} \sum_{n=1}^{\infty} \frac{1}{\epsilon^{2}}(T-t) \int_{t}^{T}\left(f_{n}(w)(s)-f_{n}(v)(s)\right)^{2} d s \\
& =\frac{1}{\epsilon^{2}}(T-t) \int_{t}^{T} \int_{0}^{\pi}(f(x, s, w(x, s))-f(x, s, v(x, s)))^{2} d x d s \\
& \leq \frac{k^{2}}{\epsilon^{2}}(T-t) \int_{t}^{T} \int_{0}^{\pi}|w(x, s)-v(x, s)|^{2} d x d s \\
& =C \frac{k^{2}}{\epsilon^{2}}(T-t)|\|w-v\||^{2} .
\end{aligned}
$$

Thus (18) holds.

Suppose that (18) holds for $m=j$. We prove that (18) holds for $m=j+1$. We have

$$
\begin{aligned}
\| G^{j+1}(w)(\cdot, t) & -G^{j+1}(v)(\cdot, t) \|^{2} \\
& \leq \frac{\pi}{2} \frac{1}{\epsilon^{2}} \sum_{n=1}^{\infty}\left[\int_{t}^{T}\left|f_{n}\left(G^{j}(w)\right)(s)-f_{n}\left(G^{j}(v)\right)(s)\right| d s\right]^{2} \\
& \leq \frac{\pi}{2} \frac{1}{\epsilon^{2}}(T-t) \int_{t}^{T} \sum_{n=1}^{\infty}\left|f_{n}\left(G^{j}(w)\right)(s)-f_{n}\left(G^{j}(v)\right)(s)\right|^{2} d s \\
& \leq \frac{1}{\epsilon^{2}}(T-t) \int_{t}^{T}\left\|f\left(\cdot, s, G^{j}(w)(\cdot, s)\right)-f\left(\cdot, s, G^{j}(v)(\cdot, s)\right)\right\|^{2} d s \\
& \leq \frac{1}{\epsilon^{2}}(T-t) k^{2} \int_{t}^{T}\left\|G^{j}(w)(\cdot, s)-G^{j}(v)(\cdot, s)\right\|^{2} d s \\
& \leq \frac{1}{\epsilon^{2}}(T-t) k^{2}\left(\frac{k}{\epsilon}\right)^{2 j} \int_{t}^{T} \frac{(T-s)^{j}}{j !} d s C^{j}|\|w-v\||^{2} \\
& \leq\left(\frac{k}{\epsilon}\right)^{2(j+1)} \frac{(T-t)^{j+1}}{(j+1) !} C^{j+1}|\|w-v\||^{2} .
\end{aligned}
$$

Therefore, by the induction principle, we have

$$
\|\| G^{m}(w)-G^{m}(v)\left\|\left|\leq\left(\frac{k}{\epsilon}\right)^{m} \frac{T^{\frac{m}{2}}}{\sqrt{m !}} \sqrt{C^{m}}\right|\right\| w-v \| \mid
$$

for all $w, v \in C\left([0, T] ; L^{2}(0, \pi)\right)$. 
We consider $G: C\left([0, T] ; L^{2}(0, \pi)\right) \rightarrow C\left([0, T] ; L^{2}(0, \pi)\right)$. There exists a positive integer $m_{0}$ such that $G^{m_{0}}$ is a contraction since $\lim _{m \rightarrow \infty}\left(\frac{k}{\epsilon}\right)^{m} \frac{T^{\frac{m}{2}} \sqrt{C^{m}}}{\sqrt{m !}}=$ 0 . It follows that the equation $G^{m_{0}}(w)=w$ has a unique solution $u_{\epsilon} \in$ $C\left([0, T] ; L^{2}(0, \pi)\right)$. In fact, one has $G\left(G^{m_{0}}\left(u^{\epsilon}\right)\right)=G\left(u^{\epsilon}\right)$. Hence $G^{m_{0}}\left(G\left(u^{\epsilon}\right)\right)=$ $G\left(u^{\epsilon}\right)$. By the uniqueness of the fixed point of $G^{m_{0}}$, one has $G\left(u^{\epsilon}\right)=u^{\epsilon}$, i.e., the equation $G(w)=w$ has a unique solution $u^{\epsilon} \in C\left([0, T] ; L^{2}(0, \pi)\right)$. From Part A, Step 1, we complete the proof of Step 2.

Step 3. The solution of the problem (4)-(6) depends continuously on $\varphi$ in $L^{2}(0, \pi)$.

Let $u$ and $v$ be two solutions of (4)-(6) corresponding to the final values $\varphi$ and $\omega$. From (7) one has in view of the inequality $(a+b)^{2} \leq 2\left(a^{2}+b^{2}\right)$

$$
\begin{aligned}
& \|u(\cdot, t)-v(\cdot, t)\|^{2} \leq \pi \sum_{n=1}^{\infty}\left(\frac{e^{-t n^{2}}}{\epsilon+e^{-T n^{2}}}\left|\varphi_{n}-\omega_{n}\right|\right)^{2} \\
& +\pi \sum_{n=1}^{\infty}\left(\int_{t}^{T} \frac{e^{-t n^{2}}}{\epsilon^{\frac{s}{T}}+e^{-s n^{2}}}\left|f_{n}(u)(s)-f_{n}(v)(s)\right| d s\right)^{2} .
\end{aligned}
$$

One has, for $s>t$ and $\alpha>0, \frac{e^{-t n^{2}}}{\alpha+e^{-s n^{2}}}=\frac{1}{\left(\alpha e^{s n^{2}}+1\right)^{\frac{t}{s}}\left(\alpha+e^{-s n^{2}}\right)^{1-\frac{t}{s}}} \leq \alpha^{\frac{t}{s}-1}$. Letting $\alpha=\epsilon, s=T$, we get

$$
\frac{e^{-t n^{2}}}{\epsilon+e^{-T n^{2}}} \leq \epsilon^{\frac{t}{T}-1}
$$

Letting $\alpha=\epsilon^{\frac{s}{T}}$, we get

$$
\frac{e^{-t n^{2}}}{\epsilon^{\frac{s}{T}}+e^{-s n^{2}}} \leq \epsilon^{\frac{t}{T}-\frac{s}{T}} .
$$

Hence, from (19) it follows that

$$
\begin{aligned}
\|u(\cdot, t)-v(\cdot, t)\|^{2} \leq & 2 \epsilon^{2\left(\frac{t}{T}-1\right)}\|\varphi-\omega\|^{2} \\
& +2 k^{2}(T-t) \epsilon^{2 \frac{t}{T}} \int_{t}^{T} \epsilon^{-2 \frac{s}{T}}\|u(\cdot, s)-v(\cdot, s)\|^{2} d s .
\end{aligned}
$$

So, we have

$$
\begin{aligned}
\epsilon^{-2\left(\frac{t}{T}\right)}\|u(\cdot, t)-v(\cdot, t)\|^{2} \leq & 2 \epsilon^{-2}\|\varphi-\omega\|^{2} \\
& +2 k^{2}(T-t) \int_{t}^{T} \epsilon^{-2 \frac{s}{T}}\|u(\cdot, s)-v(\cdot, s)\|^{2} d s .
\end{aligned}
$$

Using Gronwall's inequality we have

$$
\|u(\cdot, t)-v(\cdot, t)\| \leq 2 \epsilon^{\frac{t}{T}-1} \exp \left(k^{2}(T-t)^{2}\right)\|\varphi-\omega\| .
$$

This completes the proof of Step 3 and the proof of our theorem. 


\section{Regularization of problem (1)-(3)}

We first have a uniqueness result

Theorem 3.1. Let $\varphi, f$ be as in Theorem 2.1. Then problem (1)-(3) has at most one (weak) solution $u \in W$, where

$$
W=C\left([0, T] ; L^{2}(0, \pi)\right) \cap L^{2}\left(0, T ; H_{0}^{1}(0, \pi)\right) \cap C^{1}\left((0, T) ; L^{2}(0, \pi)\right) .
$$

Proof. Let $M>0$ be such that $\left|\frac{\partial f}{\partial z}(x, t, z)\right| \leq M$ for all $(x, t, z) \in(0, \pi) \times$ $(0, T) \times R$. Let $u_{1}(x, t)$ and $u_{2}(x, t)$ be two solutions of problem (1)-(3) such that $u_{1}, u_{2} \in W$.

Put $w(x, t)=u_{1}(x, t)-u_{2}(x, t)$. Then $w$ satisfies the equation

$$
w_{t}(x, t)-w_{x x}(x, t)=f\left(x, t, u_{1}(x, t)\right)-f\left(x, t, u_{2}(x, t)\right) .
$$

Since $f$ is Lipschizian, we have $\left(w_{t}-w_{x x}\right)^{2} \leq M^{2} w^{2}$. Now $w(0, t)=w(\pi, t)=0$ and $w(x, T)=0$. Hence by the Lees-Protter theorem ([8, p. 373]), $w=0$ which gives $u_{1}(x, t)=u_{2}(x, t)$ for all $t \in[0, T]$. The proof is completed.

Despite the uniqueness, problem (1)-(3) is still ill-posed. Hence, a regularization has to resort. We have the following result.

Theorem 3.2. Let $\varphi, f, u^{\varepsilon}$ be as in Theorem 2.1 .

a) If we can find $a u$ and a subsequence $\left(u^{\varepsilon_{j}}\right)$ in $\left(C[0, T] ; L^{2}(0, \pi)\right)$ such that

$$
u^{\varepsilon_{j}} \rightarrow u \quad \text { in } C\left([0, T] ; L^{2}(0, \pi)\right),
$$

then $u$ is the unique solution of Problem (1)-(3).

b) If problem (1)-(3) has a weak solution

$$
u \in W \text { (defined in Theorem 3.1) }
$$

which satisfies $\int_{0}^{T} \sum_{n=1}^{\infty} e^{2 s n^{2}} f_{n}^{2}(u)(s) d s<\infty$. Then

$$
\left\|u(\cdot, t)-u^{\epsilon}(\cdot, t)\right\| \leq \sqrt{M} \exp \left(\frac{3 k^{2} T(T-t)}{2}\right) \epsilon^{\frac{t}{T}}
$$

for every $t \in[0, T]$, where $M=3\|u(0)\|^{2}+6 \pi \int_{0}^{T} \sum_{n=1}^{\infty} e^{2 s n^{2}} f_{n}^{2}(u)(s) d s$ and $u^{\epsilon}$ is the unique solution of problem (4)-(6).

Proof. a) We present an outline of the proof.

The function $u^{\varepsilon_{j}}$ satisfies (4), (5) (with $\varepsilon$ replaced by $\varepsilon_{j}$ ) subject to the initial condition $u^{\varepsilon_{j}}(x, 0)=\sum_{n=1}^{\infty} \varphi_{n}^{j} \sin n x$ and $u(x, 0)=\sum_{n=1}^{\infty} u_{n}(0) \sin n x$. One gets (see [5])

$$
u^{\varepsilon_{j}}(x, t)=\sum_{n=1}^{\infty}\left[e^{-t n^{2}} \varphi_{n}^{j}+\int_{0}^{t} \frac{e^{-t n^{2}}}{\varepsilon_{j}^{s / T}+e^{-s n^{2}}} f_{n}\left(u^{\varepsilon_{j}}\right) d s\right] \sin n x .
$$


Letting $\varepsilon \downarrow 0$, we shall get

$$
u(x, t)=\sum_{n=1}^{\infty}\left(e^{-t n^{2}} u_{n}(0)+\int_{0}^{t} e^{-(t-s) n^{2}} f_{n}(u) d s\right) \sin n x .
$$

On the other hand, letting $\varepsilon \downarrow 0$ in (6), we get $u(x, T)=\varphi(x)$. Hence $u$ is the solution of problem (1)-(3) as desired.

b) The exact solution $u$ satisfies

$$
\begin{aligned}
u(x, t) & =\sum_{n=1}^{\infty}\left(e^{-(t-T) n^{2}} \varphi_{n}-\int_{t}^{T} e^{-(t-s) n^{2}} f_{n}(u)(s) d s\right) \sin n x \\
u(x, T) & =\sum_{n=1}^{\infty}\left(e^{-T n^{2}} u_{n}(0)+\int_{0}^{T} e^{-(T-s) n^{2}} f_{n}(u)(s) d s\right) \sin n x=\sum_{n=1}^{\infty} \varphi_{n} \sin n x
\end{aligned}
$$

where we recall $u_{n}(0)=\frac{2}{\pi}\langle u(x, 0), \sin n x\rangle$ (see [5]). Hence

$$
e^{-T n^{2}} u_{n}(0)+\int_{0}^{T} e^{-(T-s) n^{2}} f_{n}(u)(s) d s=\varphi_{n} .
$$

From (7), (22) and (23), we get

$$
\begin{aligned}
\left|u_{n}(t)-u_{n}^{\epsilon}(t)\right|= & \mid \frac{\epsilon e^{-t n^{2}}}{e^{-T n^{2}}\left(\epsilon+e^{-T n^{2}}\right)} \varphi_{n}-\int_{t}^{T} \frac{\epsilon^{\frac{s}{T}} e^{-t n^{2}}}{e^{-s n^{2}}\left(\epsilon^{\frac{s}{T}}+e^{-s n^{2}}\right)} f_{n}(u)(s) d s \\
& -\int_{t}^{T} \frac{e^{-t n^{2}}}{\epsilon^{\frac{s}{T}}+e^{-s n^{2}}}\left(f_{n}(u)(s)-f_{n}\left(u^{\epsilon}\right)(s)\right) d s \mid \\
\leq & \mid \frac{\epsilon e^{-t n^{2}}}{\epsilon+e^{-T n^{2}}} u_{n}(0)+\int_{0}^{T} \frac{\epsilon e^{-t n^{2}}}{e^{-s n^{2}}\left(\epsilon+e^{-T n^{2}}\right)} f_{n}(u)(s) d s \\
& -\int_{t}^{T} \frac{\epsilon^{\frac{s}{T}} e^{-t n^{2}}}{e^{-s n^{2}}\left(\epsilon^{\frac{s}{T}}+e^{-s n^{2}}\right)} f_{n}(u(s) d s \mid \\
& +\int_{t}^{T} \frac{e^{-t n^{2}}}{\epsilon^{\frac{s}{T}}+e^{-s n^{2}}}\left|f_{n}(u)(s)-f_{n}\left(u^{\epsilon}\right)(s)\right| d s .
\end{aligned}
$$

From (20), (21) and (24), we have

$$
\begin{aligned}
\mid u_{n}(t)- & u_{n}^{\epsilon}(t) \mid \\
\leq & \epsilon \cdot \epsilon^{\frac{t}{T}-1}\left|u_{n}(0)\right|+\int_{0}^{T} \epsilon \cdot \epsilon^{\frac{t}{T}}-1\left|\frac{f_{n}(u)(s)}{e^{-s n^{2}}}\right| d s+\int_{t}^{T} \epsilon^{\frac{s}{T}} \cdot \epsilon^{\frac{t}{T}}-\frac{s}{T}\left|\frac{f_{n}(u)(s)}{e^{-s n^{2}}}\right| d s \\
& +\int_{t}^{T} \epsilon^{\frac{t}{T}-\frac{s}{T}}\left|f_{n}(u)(s)-f_{n}\left(u^{\epsilon}\right)(s)\right| d s \\
\leq & \epsilon^{\frac{t}{T}}\left|u_{n}(0)\right|+2 \epsilon^{\frac{t}{T}} \int_{0}^{T}\left|\frac{f_{n}(u)(s)}{e^{-s n^{2}}}\right| d s+\epsilon^{\frac{t}{T}} \int_{t}^{T} \epsilon^{-\frac{s}{T}}\left|f_{n}(u)(s)-f_{n}\left(u^{\epsilon}\right)(s)\right| d s .
\end{aligned}
$$


We have in view of the inequality $(a+b+c)^{2} \leq 3\left(a^{2}+b^{2}+c^{2}\right)$

$$
\begin{aligned}
\left\|u(\cdot, t)-u^{\epsilon}(\cdot, t)\right\|^{2}= & \frac{\pi}{2} \sum_{n=1}^{\infty}\left|u_{n}(t)-u_{n}^{\epsilon}(t)\right|^{2} \\
\leq & \frac{3 \pi}{2} \sum_{n=1}^{\infty} \epsilon^{2 \frac{t}{T}}\left|u_{n}(0)\right|^{2}+6 \pi \sum_{n=1}^{\infty} \epsilon^{2 \frac{t}{T}}\left(\int_{0}^{T}\left|\frac{1}{e^{-s n^{2}}} f_{n}(u)(s)\right| d s\right)^{2} \\
& +\frac{3 \pi}{2} \sum_{n=1}^{\infty} \epsilon^{2 \frac{t}{T}}\left(\int_{t}^{T} \epsilon^{-\frac{s}{T}}\left|f_{n}(u)(s)-f_{n}\left(u^{\epsilon}\right)(s)\right| d s\right)^{2} \\
\leq & 3 \epsilon^{2 \frac{t}{T}}\|u(0)\|^{2}+6 \pi T \epsilon^{2 \frac{t}{T}} \int_{0}^{T} \sum_{n=1}^{\infty} e^{2 s n^{2}} f_{n}^{2}(u)(s) d s \\
& +3(T-t) \epsilon^{2 \frac{t}{T}} \int_{t}^{T} \epsilon^{-2 \frac{s}{T}}\left\|f(\cdot, s, u(\cdot, s))-f\left(\cdot, s, u^{\epsilon}(\cdot, s)\right)\right\|^{2} d s \\
\leq & \epsilon^{2 \frac{t}{T}}\left(3\|u(0)\|^{2}+6 \pi T \int_{0}^{T} \sum_{n=1}^{\infty} e^{2 s n^{2}} f_{n}(u(s))^{2} d s\right. \\
& \left.+3 k^{2} T \int_{t}^{T} \epsilon^{-2 \frac{s}{T}}\left\|u(\cdot, s)-u^{\epsilon}(\cdot, s)\right\|^{2} d s\right) .
\end{aligned}
$$

Hence

$$
\epsilon^{-2 \frac{t}{T}}\left\|u(\cdot, t)-u^{\epsilon}(\cdot, t)\right\|^{2} \leq M+3 k^{2} T \int_{t}^{T} \epsilon^{-2 \frac{s}{T}}\left\|u(\cdot, s)-u^{\epsilon}(\cdot, s)\right\|^{2} d s,
$$

where $M=3\|u(0)\|^{2}+6 \pi T \int_{0}^{T} \sum_{n=1}^{\infty} e^{2 s n^{2}} f_{n}^{2}(u)(s) d s$. Using Gronwall's inequality, we get

$$
\epsilon^{-2 \frac{t}{T}}\left\|u(\cdot, t)-u^{\epsilon}(\cdot, t)\right\|^{2} \leq M e^{3 k^{2} T(T-t)} .
$$

This completes the proof of Theorem 3.2.

\section{Remark 3.3.}

1. From Part a), we conclude that if problem (1)-(3) does not have any exact solution $u \in W$, then one has

$$
\lim _{\varepsilon \downarrow 0} \inf \left\{\max _{0 \leq t \leq T}\left\|u^{\varepsilon}(\cdot, t)-\psi(\cdot, t)\right\|\right\}>0
$$

for every $\psi \in C\left([0, T] ; L^{2}(0, \pi)\right)$.

2. If $f(x, t, u) \equiv 0$, we have the linear homogeneous problem, the error estimate is as in [2].

3. From (23), one has

$$
u_{n}(0)=\varphi_{n} e^{T n^{2}}-\int_{0}^{T} e^{s n^{2}} f_{n}(u)(s) d s .
$$


If $\sum_{n=1}^{\infty} \varphi_{n}^{2} e^{2 T n^{2}}<\infty$, then $\sum_{n=1}^{\infty}\left(\int_{0}^{T} e^{s n^{2}} f_{n}(u)(s) d s\right)^{2}<\infty$. Hence the assumptions of $f$ in Theorem 3.2 are reasonable.

One has

Theorem 3.4. Let $\varphi, f$ be as in Theorem 2.1 and let $u \in W$ be a solution of problem $(1)-(3)$ such that $\frac{\partial u}{\partial t} \in L^{2}\left((0, T) ; L^{2}(0, \pi)\right)$ and $\int_{0}^{T} \sum_{n=1}^{\infty} e^{2 s n^{2}} f_{n}^{2}(u)(s) d s<$ $\infty$. Then for all $\epsilon>0$ there exists a $t_{\epsilon}$ such that

$$
\left\|u(\cdot, 0)-u^{\epsilon}\left(\cdot, t_{\epsilon}\right)\right\| \leq \sqrt[4]{8} C \sqrt[4]{T}\left(\ln \left(\frac{1}{\epsilon}\right)\right)^{-\frac{1}{4}}
$$

where

$$
C=\max \left\{\exp \left(\frac{3 k^{2} T^{2}}{2}\right)\left(3\left\|u_{0}(\cdot, 0)\right\|^{2}+6 \pi T \int_{0}^{T} \sum_{n=1}^{\infty} e^{2 s n^{2}} f_{n}^{2}(u)(s) d s\right)^{\frac{1}{2}}, N\right\}
$$

and

$$
N=\left(\int_{0}^{T}\left\|\frac{\partial u}{\partial t}(\cdot, s)\right\|^{2} d s\right)^{\frac{1}{2}}
$$

Proof. We have $u(x, t)-u(x, 0)=\int_{0}^{t} \frac{\partial u}{\partial s}(x, s) d s$. It follows that

$$
\|u(\cdot, 0)-u(\cdot, t)\|^{2} \leq t \int_{0}^{t}\left\|\frac{\partial u}{\partial t}(\cdot, s)\right\|^{2} d s=N^{2} t
$$

Using Theorem 3.2 and (25)-(26), we have

$$
\left\|u(\cdot, 0)-u^{\epsilon}(\cdot, t)\right\| \leq\|u(\cdot, 0)-u(\cdot, t)\|+\left\|u(\cdot, t)-u^{\epsilon}(\cdot, t)\right\| \leq C\left(\sqrt{t}+\epsilon^{\frac{t}{T}}\right) .
$$

For every $\epsilon$, there exists $t_{\epsilon}$ such that $\sqrt{t_{\epsilon}}=\epsilon^{\frac{t_{\epsilon}}{T}}$, i.e., $\frac{\ln t_{\epsilon}}{t_{\epsilon}}=\frac{2 \ln \epsilon}{T}$. Using inequality $\ln t>-\frac{1}{t}$ for every $t>0$, we get

$$
\left\|u(\cdot, 0)-u^{\epsilon}\left(\cdot, t_{\epsilon}\right)\right\| \leq \sqrt[4]{8} C \sqrt[4]{T}\left(\ln \left(\frac{1}{\epsilon}\right)\right)^{-\frac{1}{4}} .
$$

This completes the proof of Theorem 3.4.

Remark 3.5. Using the Galerkin method (see, e.g., [9]), we can show that the assumption on $u_{t}$ holds if $u(\cdot, 0) \in H_{0}^{1}(0, \pi)$.

In the case of nonexact data, one has 
Theorem 3.6. Let $\varphi, f$ be as in Theorem 2.1. Assume that the exact solution $u$ of (1)-(3) corresponding to $\varphi$ satisfies

$$
u \in W, \quad \frac{\partial u}{\partial t} \in L^{2}\left((0, T) ; L^{2}(0, \pi)\right)
$$

and $\int_{0}^{T} \sum_{n=1}^{\infty} e^{2 s n^{2}} f_{n}^{2}(u)(s) d s<\infty$. Let $\varphi_{\epsilon} \in L^{2}(0, \pi)$ be a measured data such that $\left\|\varphi_{\epsilon}-\varphi\right\| \leq \epsilon$. Then there exists a function $u^{\epsilon}$ satisfying

$$
\begin{aligned}
\left\|u^{\epsilon}(\cdot, t)-u(\cdot, t)\right\| & \leq(2+\sqrt{M}) \exp \left(\frac{3 k^{2} T(T-t)}{2}\right) \epsilon^{\frac{t}{T}}, \quad \text { for every } t \in(0, T) \\
\left\|u^{\epsilon}(\cdot, 0)-u(\cdot, 0)\right\| & \leq \sqrt[4]{8} \sqrt[4]{T}\left(\ln \left(\frac{1}{\epsilon}\right)\right)^{-\frac{1}{4}}\left(\exp \left(k^{2} T^{2}\right)+C\right)
\end{aligned}
$$

where $M=3\|u(\cdot, 0)\|^{2}+6 \pi T \int_{0}^{T} \sum_{n=1}^{\infty} e^{2 s n^{2}} f_{n}^{2}(u)(s) d s$, and $C$ is defined in (25)-(26).

Proof. Let $v^{\epsilon}$ be the solution of problem (4)-(6) corresponding to $\varphi$ and let $w^{\epsilon}$ be the solution of problem (4)-(6) corresponding to $\varphi_{\epsilon}$, where $\varphi, \varphi_{\epsilon}$ are in right hand side of (6).

Using Theorem 3.4, there exists a $t_{\epsilon}$ such that

$$
\sqrt{t_{\epsilon}}=\epsilon^{\frac{t_{\epsilon}}{T}}
$$

and

$$
\left\|v^{\epsilon}\left(\cdot, t_{\epsilon}\right)-v(\cdot, 0)\right\| \leq \sqrt[4]{8} C \sqrt[4]{T}\left(\ln \left(\frac{1}{\epsilon}\right)\right)^{-\frac{1}{4}}
$$

Put

$$
u^{\epsilon}(\cdot, t)= \begin{cases}w^{\epsilon}(\cdot, t), & 0<t<T \\ w^{\epsilon}\left(\cdot, t_{\epsilon}\right), & t=0 .\end{cases}
$$

Using Theorem 3.2 and Step 3 in Theorem 2.1, we get

$$
\begin{aligned}
\left\|u^{\epsilon}(\cdot, t)-u(\cdot, t)\right\| & \leq\left\|w^{\epsilon}(\cdot, t)-v^{\epsilon}(\cdot, t)\right\|+\left\|v^{\epsilon}(\cdot, t)-u(\cdot, t)\right\| \\
& \leq(2+\sqrt{M}) \exp \left(\frac{3 k^{2} T(T-t)}{2}\right) \epsilon^{\frac{t}{T}},
\end{aligned}
$$

for every $t \in(0, T)$. From (27)-(28) and Step 3 in Theorem 2.1, we have

$$
\begin{aligned}
\left\|u^{\epsilon}(\cdot, 0)-u(\cdot, 0)\right\| & \leq\left\|w^{\epsilon}\left(\cdot, t_{\epsilon}\right)-v^{\epsilon}\left(\cdot, t_{\epsilon}\right)\right\|+\left\|v^{\epsilon}\left(\cdot, t_{\epsilon}\right)-u(\cdot, 0)\right\| \\
& \leq 2 \epsilon^{\frac{t_{\epsilon}}{T}} \exp \left(k^{2} T^{2}\right)+\sqrt[4]{8} C \sqrt[4]{T}\left(\ln \left(\frac{1}{\epsilon}\right)\right)^{-\frac{1}{4}} \\
& \leq \sqrt[4]{8} \sqrt[4]{T}\left(\ln \left(\frac{1}{\epsilon}\right)\right)^{-\frac{1}{4}}\left(\exp \left(k^{2} T^{2}\right)+C\right),
\end{aligned}
$$

where $C$ is defined in (25)-(26). This completes the proof of Theorem 3.6. 


\section{A numerical experiment}

We consider the equation

$$
-u_{x x}+u_{t}=f(u)+g(x, t),
$$

where $g(x, t)=2 e^{t} \sin x-e^{4 t} \sin ^{4} x, u(x, 1)=\varphi_{0}(x) \equiv e \sin x$ and

$$
f(u)= \begin{cases}u^{4}, & u \in\left[-e^{10}, e^{10}\right] \\ -\frac{e^{30}}{e-1} u+\frac{e^{41}}{e-1}, & u \in\left(e^{10}, e^{11}\right] \\ \frac{e^{30}}{e-1} u+\frac{e^{41}}{e-1}, & u \in\left(-e^{11},-e^{10}\right] \\ 0, & |u|>e^{11}\end{cases}
$$

The exact solution of the equation is $u(x, t)=e^{t} \sin x$. Especially, $u\left(x, \frac{99}{100}\right) \equiv$ $u(x)=\exp \left(\frac{99}{100}\right) \sin x$. Let $\varphi_{\epsilon}(x) \equiv \varphi(x)=(\epsilon+1) e \sin x$. We have

$$
\left\|\varphi_{\epsilon}-\varphi\right\|_{2}=\left(\int_{0}^{\pi} \epsilon^{2} e^{2} \sin ^{2} x d x\right)^{\frac{1}{2}}=\epsilon e\left(\frac{\pi}{2}\right)^{\frac{1}{2}} .
$$
form:

We find the regularized solution $u_{\epsilon}\left(x, \frac{99}{100}\right) \equiv u_{\epsilon}(x)$ having the following

$$
u_{\epsilon}(x)=v_{m}(x)=w_{1, m} \sin x+w_{2, m} \sin 2 x+w_{3, m} \sin 3 x,
$$

where $v_{1}(x)=(\epsilon+1) e \sin x, w_{1,1}=(\epsilon+1) e, w_{2,1}=0, w_{3,1}=0$ and

$$
\left\{\begin{array}{l}
w_{i, m+1}=\frac{e^{-t_{m+1} i^{2}}}{\epsilon+e^{-t_{m} i^{2}}} w_{i, m}-\frac{2}{\pi} \int_{t_{m+1}}^{t_{m}} \frac{e^{-t_{m+1} i^{2}}}{\epsilon^{\frac{s}{t_{m}}}+e^{-s i^{2}}}\left(\int_{0}^{\pi}\left(v_{m}^{4}(x)+g(x, s)\right) \sin i x d x\right) d s \\
t_{m}=1-a m, \quad a=\frac{1}{40000}, \quad m=1,2, \ldots, 4000, \quad i=1,2,3 .
\end{array}\right.
$$

Put $a_{\epsilon}=\left\|u_{\epsilon}-u\right\|$ the error between the regularization solution $u_{\epsilon}$ and the exact solution $u$. Letting $\epsilon=\epsilon_{1}=10^{-5}, \epsilon=\epsilon_{2}=10^{-7}, \epsilon=\epsilon_{3}=10^{-11}$, numerical results are given as follows.

\begin{tabular}{|c|c|c|}
\hline$\epsilon$ & $u_{\epsilon}$ & $a_{\epsilon}$ \\
\hline$\epsilon_{1}=10^{-5}$ & $2.430605996 \sin x-0.000171846090 \sin 3 x$ & 0.32664942510 \\
\hline$\epsilon_{2}=10^{-7}$ & $2.646937077 \sin x-0.002178680692 \sin 3 x$ & 0.05558566020 \\
\hline$\epsilon_{3}=10^{-11}$ & $2.649052245 \sin x-0.004495263004 \sin 3 x$ & 0.05316693437 \\
\hline
\end{tabular}


Acknowledgement. The authors wish to thank the referee for their valuable criticisms and suggestions, leading to the present improved version of our paper.

\section{References}

[1] Alekseeva, S. M. and Yurchuk, N. I., The quasi-reversibility method for the problem of the control of an initial condition for the heat equation with an integral boundary condition. Diff. Equations 34 (1998)(4), 493 - 500.

[2] Ames, K. A., Clark, G. W., Epperson, J. F., Oppenheimer, S. F., A comparison of regularizations for an ill-posed problem. Math. Comp. 67 (1998), no.224, $1451-1471$.

[3] Ames, K. A. and Payne, L. E., Continuous dependence on modeling for some well-posed perturbations of the backward heat equation. J. Inequal. Appl. 3 (1999)(1), $51-64$.

[4] Clark, G. and Oppenheimer, C., Quasireversibility methods for non-well-posed problem. Electron. J. Diff. Equations 8 (1994), 1 - 9.

[5] Colton, D., Partial Differential Equations. New York: Random House 1988.

[6] Denche, M. and Bessila, K., Quasi-boundary value method for non-well posed problem for a parabolic equation with integral boundary condition. Math. Probl. Eng. 7 (2001)(2), 129 - 145.

[7] Lattes, R. and Lions, J. L., Methode de Quasi-Reversibilité et Applications. Paris: Dunod 1967.

[8] Lees, M. and Protter, M. H., Unique continuation for parabolic differential equations and inequalities. Duke Math. J. 28 (1961), 369 - 382.

[9] Lions, J. L., Quelques méthodes de résolution des problèmes aux limites nonlinéaires (in French). Paris: Dunod, Gauthier-Villars 1969.

[10] Miller, K., Stabilized quasi-reversibility and other nearly-best-possible methods for non-well-posed problems. Symposium on Non-Well-Posed Problems and Logarithmic Convexity (Heriot-Watt Univ., Edinburgh 1972; ed.: R. J. Knops). Lecture Notes Math. 316. Berlin: Springer 1973, pp. $161-176$.

[11] Quan, P. H. and Dung, N., A backward nonlinear heat equation: regularization with error estimates, Appl. Anal. 84 (2005)(4), 343 - 355.

[12] Showalter, R. E., Quasi-reversibility of first and second order parabolic evolution equations. Improperly Posed Boundary Value Problems (Conf., Univ. New Mexico, Albuquerque, N. M., 1974; eds.: A. Carasso and A. P. Stone). Res. Notes Math. 1. London: Pitman 1975, pp. $76-84$.

Received May 29, 2005; revised February 13, 2006 